\title{
Immunohistochemical Expression of Cytokeratin-19 in Thyroid Nodules and Its Correlation With Histopathology
}

\author{
Suriyaprabha Palanisamy ${ }^{1 *}$, Dhanalakshmi Arumugam² and Sudha $\mathrm{Boj}^{2}$ \\ ${ }^{1}$ Department of Pathology, Theni Medical College, Theni, Tamilnadu. India \\ ${ }^{2}$ Department of Pathology, Coimbatore Medical College, Coimbatore, Tamilnadu, India
}

\section{ABSTRACT}

Background: Thyroid neoplasms constitute the most commonly occurring endocrine tumors worldwide. They commonly occur between 30-60 years of age and affect both sexes. Papillary carcinoma is the most common malignant tumor constituting $80-85 \%$ of all the thyroid carcinomas followed by follicular carcinoma comprising 10-15\%. Diagnosis becomes challenging when typical features are not seen or when overlapping features are present. In such cases immunohistochemistry can be done to make correct diagnosis.

Methods: In this study we observed the immunohistochemical expression of Cytokeratin19 in 30 thyroidectomy specimens which included classic papillary carcinoma (11 cases), follicular variant of papillary carcinoma ( 2 cases), follicular adenoma (15 cases) and follicular carcinoma (4 cases). The study period was one year. The scoring was given from $0-3+$ based on the percentage of cells showing membrane and cytoplasmic positivity and intensity of staining.

Result: The staining results of Cytokeratin19 showed diffuse and strong 3+ positivity in 8/11 cases of papillary carcinoma and its follicular variant, 2 cases showed $2+$ positivity and one case with $1+$ positivity. Majority of the cases of follicular adenoma (13/15 cases) and follicular carcinoma (3/4 cases) showed negative staining. Only 2 cases of follicular adenoma and 1 case of follicular carcinoma showed focal weak positivity.

Conclusion: Cytokeratin19 was found to be a sensitive and specific marker in diagnosing papillary carcinoma and its follicular variant. But it does not differentiate follicular neoplasms. Hence a good panel of markers is essential in arriving at exact diagnosis of thyroid neoplasms in challenging cases.

Keywords: Papillary Carcinoma, Follicular Adenoma, Follicular Carcinoma, Cytokeratin19.

\section{Introduction}

Thyroid neoplasms constitute the most commonly occurring endocrine tumors worldwide and commonly occur between 30-60 years of age. ${ }^{[1]}$ About $4 \%$ to $8 \%$ of adult women and $1 \%$ to $2 \%$ of adult men present with thyroid nodules. Majority of the thyroid nodules are benign with malignant nodules comprising only $10 \%$. Papillary carcinoma is the most common malignant tumor constituting $80-85 \%$ of all the thyroid carcinomas followed by follicular carcinoma comprising $10-15 \% .{ }^{[2]}$ But the mortality rate is only $6.5 \%$. Diagnosis becomes challenging when typical features are not seen or when overlapping features are present. In such cases immunohistochemistry (IHC) can be done to make correct diagnosis.

In this study we have analysed the usefulness of cytokeratin19 in differentiating thyroid neoplasms by grading the intensity of staining in membrane and cytoplasm of cells and correlated it with the histopathology. Cytokeratin19 is a low molecular weight intermediate filament protein of $40 \mathrm{KDa}$ and belongs to keratin family.
[3] This was proved to be a sensitive and specific marker of papillary carcinoma but failed to distinguish follicular neoplasms.

\section{Materials and Methods}

This is a prospective study undertaken in the Department of Pathology for a period of 1 year from June 2014- May 2015. A total of 30 cases of thyroidectomy specimens received were analysed. This study was approved by our institutional ethics committee.

Inclusion Criteria: is all thyroidectomy specimens done for solitary and multiple neoplastic nodules.

Exclusion criteria: Multinodular goiter and Toxic goiter.

Among the total thyroidectomy specimens that were received in the department of Pathology in our hospital during the study period, 30 cases were taken into study as per the inclusion criteria and were evaluated further .

All those 30 thyroidectomy specimens selected were histologically evaluated and categorized as following 
- Follicular adenoma

- Follicular carcinoma.

- Papillary carcinoma (one with lymph node metastasis)

- Follicular variant of papillary carcinoma.

Follicular carcinoma is differentiated from follicular adenoma by widespread invasion into thyroid parenchyma, capsular invasion and/ or vascular invasion. These tumors may lack complete encapsulation and may present with distant metastasis at the initial presentation. ${ }^{[4,5]}$

Papillary carcinoma is diagnosed based on papillary pattern and characteristic nuclear features like ovoid ground glass nuclei with fine chromatin, nuclear grooves, nuclear pseudoinclusions, nuclear crowding and overlapping with or without psammoma body. ${ }^{[6]}$

IHC was done by utilizing a monoclonal anticytokeratin 19 antibody (clone -RCK 108), a mouse monoclonal antibody. Skin samples were used as positive control. The antigen retrieval was done by microwave method using citrate buffer at $\mathrm{pH}$ 6. Diaminbenzidine tetrachloride (DAB) was used as a chromogen and hematoxylin as a counterstain.

Positive staining refers to diffuse staining of membrane and cytoplasm. Intensity of positive staining was graded from 0 to $3+$. Scoring was done based on the intensity of staining and percentage of cells expressing cytokeratin 19 as shown below. ${ }^{[7]}$

\begin{tabular}{|l|l|l|}
\hline S.No & Grading & $\begin{array}{l}\text { Percentage of cells } \\
\text { expressing CK19 positivity }\end{array}$ \\
\hline 1. & 0 (negative) & No positively staining cells \\
\hline 2. & $\begin{array}{l}1+(\text { focally } \\
\text { positive) }\end{array}$ & $\begin{array}{l}<25 \% \text { of positively staining } \\
\text { cells }\end{array}$ \\
\hline 3. & $2+$ (positive) & $\begin{array}{l}25-50 \% \text { of positively } \\
\text { staining cells }\end{array}$ \\
\hline 4. & $\begin{array}{l}3+(\text { diffusely } \\
\text { positive })\end{array}$ & $\begin{array}{l}>50 \% \text { of positively staining } \\
\text { cells }\end{array}$ \\
\hline
\end{tabular}

\section{Results}

A total of 30 cases of thyroidectomy specimens were taken for this study. Histomorphological features and immunohistochemical expression pattern of cytokeratin 19 in thyroid nodules were studied, analysed and compared with literature.

In the present study the age distribution of thyroid neoplasms was most common in the age group between $41-50$ years, comprising about $40 \%$ ( 12 cases) of the total cases (Table 1). Thyroid nodules were more common among females constituting about $90 \%$ (27 cases out of 30 ).Female to male ratio was $3: 1$ in this study.

Comparing the incidence of different thyroid neoplasms, follicular adenoma constituted the majority of cases (50\%), followed by papillary carcinoma (37\%- classic type- $30 \%$, follicular variant- $7 \%$ ) and follicular carcinoma $-13 \%$ (Table 2).

The intensity of staining of cytokeratin 19 in various thyroid neoplasms were analysed and tabulated (Table 3, chart 1). In the present study, staining intensity of cytokeratin 19 in classic papillary carcinoma showed strong and diffuse positivity in membrane and cytoplasm of cells i.e. 3+ staining in 8 cases comprising $89 \%$, with only $11 \%$ of cases showing moderate or $2+$ staining (Fig 1, Fig 2). In follicular variant of papillary carcinoma out of 2 cases, one showed $2+$ positivity (Fig 3), other case showed $1+$ positivity Thus all cases of papillary carcinoma showed cytokeratin 19 expression i.e. 100\%.

In follicular carcinoma cytokeratin19 is weakly positive i.e. $1+$ in only one case while other cases were negative for cytokeratin19 (Fig 4).

In follicular adenoma $1+$ weak and focal positivity was obtained in $13 \%$ of cases only (Fig 5). $87 \%$ of cases were negative for cytokeratin 19 .

Chi square test showed statistically significant $p$ value of $<0.001$ from the above variables.

Table 1

\begin{tabular}{|c|c|c|}
\hline Age in years & No. of cases & Percentage \\
\hline$<30$ & 8 & $27 \%$ \\
\hline $31-40$ & 8 & $27 \%$ \\
\hline $41-50$ & 12 & $40 \%$ \\
\hline $51-60$ & 2 & $6 \%$ \\
\hline Total & 30 & $\mathbf{1 0 0 \%}$ \\
\hline
\end{tabular}

Table 2: distribution of different thyroid neoplasms:

\begin{tabular}{|c|c|c|}
\hline Histopathological Diagnosis & No. of cases & Percentage \\
\hline Papillary Carcinoma & 11 & $37 \%$ \\
\hline Classic type & 9 & $30 \%$ \\
\hline Follicular variant & 2 & $7 \%$ \\
\hline Follicular Carcinoma & 4 & $13 \%$ \\
\hline Follicular Adenoma & 15 & $50 \%$ \\
\hline
\end{tabular}


TABLE 3: Scoring Of Cytokeratin19 Expression In Thyroid Nodules.

\begin{tabular}{|c|c|c|c|c|c|}
\hline \multirow{2}{*}{ Staining of CK 19} & \multicolumn{5}{|c|}{ HPE DIAGNOSIS } \\
\hline & PC & FC & FA & PCFV & TOTAL \\
\hline 0 & 0 & 3 & 13 & 0 & 16 \\
\hline $1+$ & 0 & 1 & 2 & 1 & 4 \\
\hline $2+$ & 1 & 0 & 0 & 1 & 2 \\
\hline $3+$ & 8 & 0 & 0 & 0 & 8 \\
\hline Total & 9 & 4 & 15 & 2 & 30 \\
\hline
\end{tabular}

(Note: 0: no cells are positive; $1+:<25 \%$ of cells are positive; $2+: 25 \%-50 \%$ of cells are positive; $3+:>50 \%$ of cells are positive, PC: Papillary carcinoma, PCFV: Papillary carcinoma - Follicular variant, FA: Follicular adenoma, FC: Follicular Carcinoma, HPE: Histopathology)

TABLE 4: Statistical Analysis Datas Of Cytokeratin19 Staining In Thyroid Nodules.

\begin{tabular}{|c|c|c|}
\hline Thyroid tumors & Sensitivity & specificity \\
\hline Classic papillary carcinoma & $100 \%$ & $84 \%$ \\
\hline Papillary carcinoma-Follicular variant & $100 \%$ & $57 \%$ \\
\hline Follicular carcinoma & $25 \%$ & $50 \%$ \\
\hline Follicular adenoma & $13 \%$ & $87 \%$ \\
\hline
\end{tabular}

TABLE 5: Comparison Of Results Of Cytokeratin19 Expression In Various Studies With Our Study.

\begin{tabular}{|l|c|c|c|}
\hline \multirow{2}{*}{ Study name } & \multicolumn{2}{|c|}{ Percentage of cases with positive cytokeratin19 expression } \\
\cline { 2 - 4 } & Follicular adenoma & Follicular carcinoma & $\begin{array}{c}\text { Papillary carcinoma and } \\
\text { follicular variant }\end{array}$ \\
\hline Hanan Alsaeid Alshenawy & $57 \%$ & $53 \%$ & $100 \%$ \\
\hline Husain A Saleh et al & $30 \%$ & - & $85 \%$ \\
\hline K.Y Lam et al & - & $68 \%$ & - \\
\hline Dina El Demellawy et al & - & - & $85 \%$ \\
\hline Our study & $13 \%$ & $50 \%$ & $100 \%$ \\
\hline
\end{tabular}

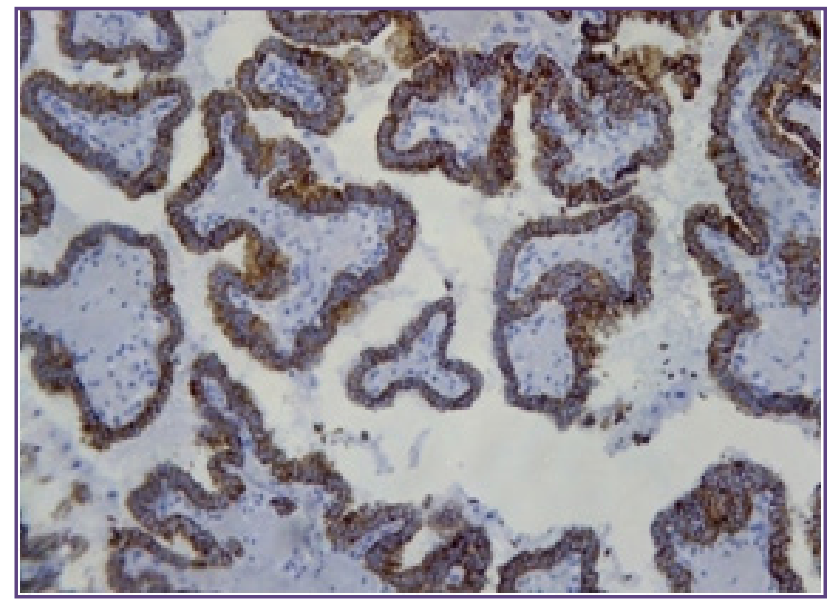

Fig. 1: Diffuse 3+ positivity of cytokeratin19 in papillary carcinoma (10X).

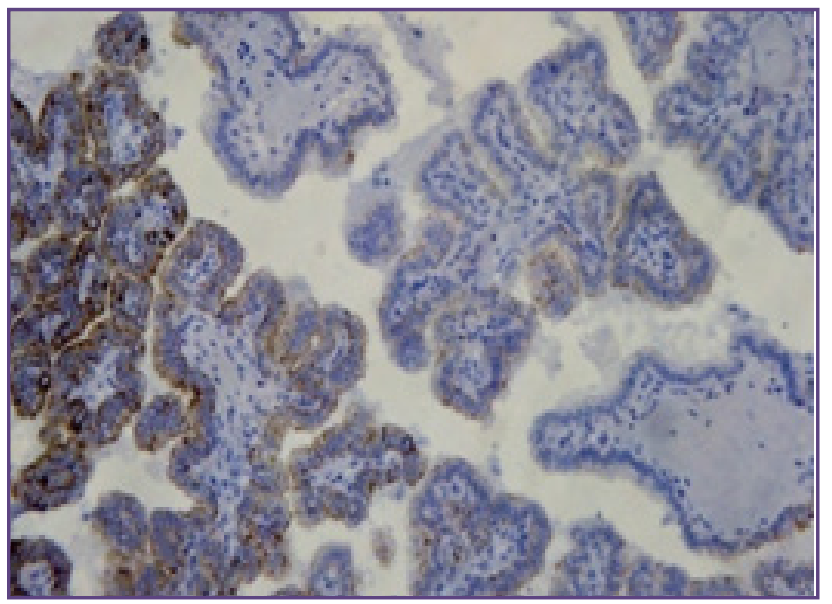

Fig. 2: 2+ positivity of Cytokeratin19 in papillary carcinoma (10X) 


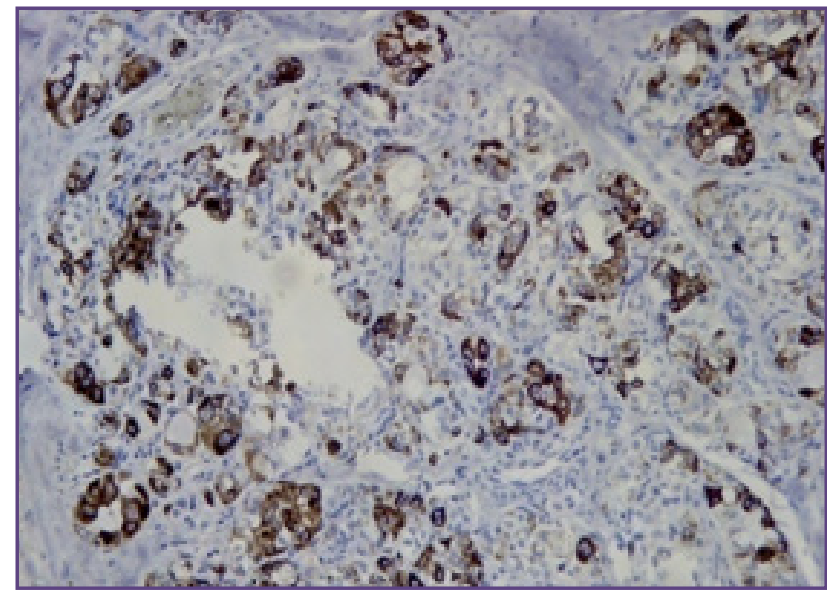

Fig. 3: Follicular variant of papillary carcinoma showing $2+$ positivity with cytokeratin19 (10X).

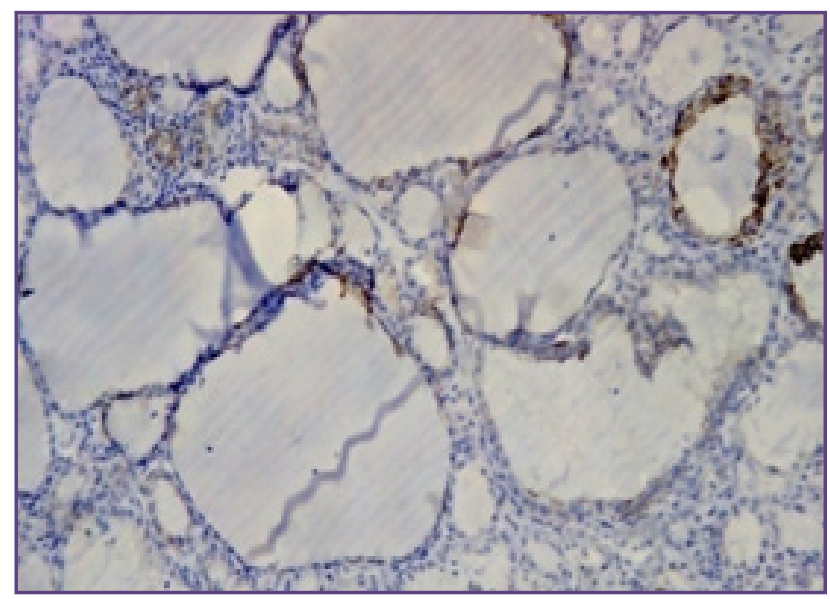

Fig. 5: Follicular adenoma showing focal 1+ positivity with cytokeratin19 (10X).

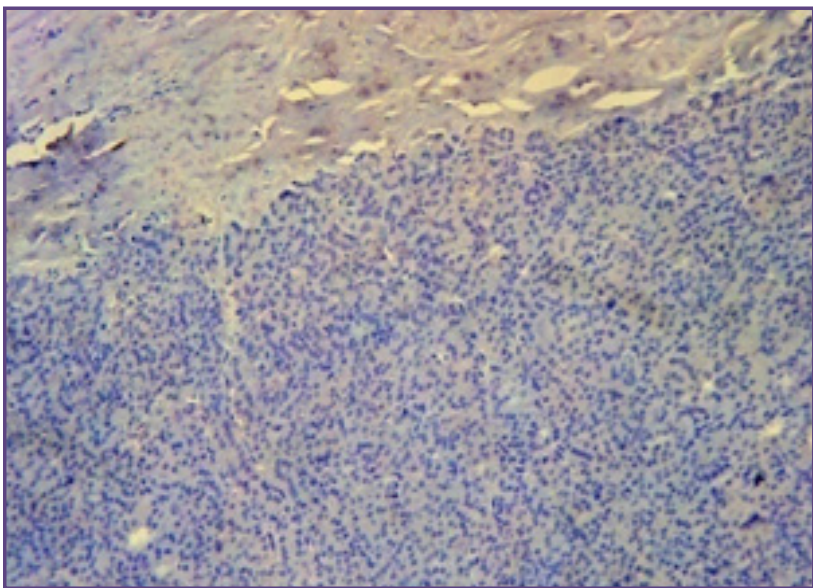

Fig. 4: Follicular carcinoma showing negative staining with cytokeratin19.

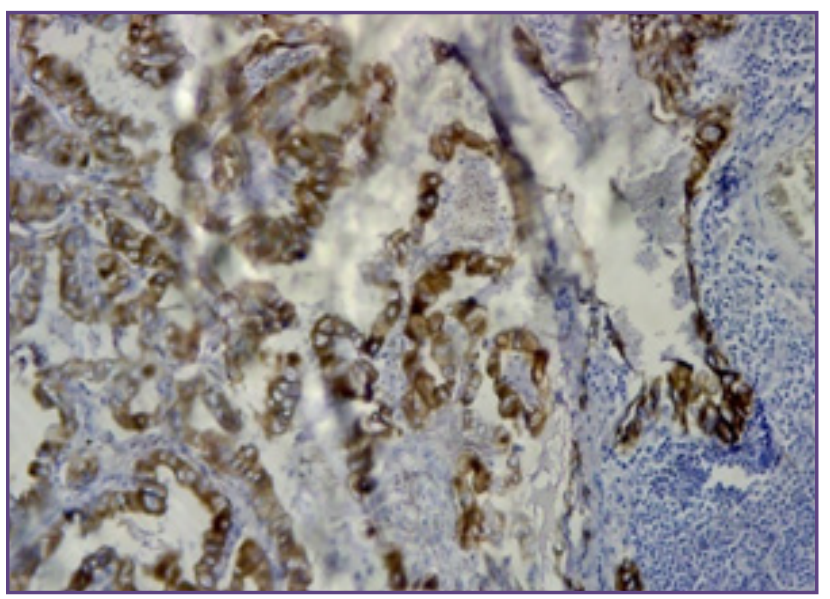

Fig. 6: Diffuse 3+ positivity of cytokeratin19 in metastatic papillary carcinoma deposits in lymph node (10X).

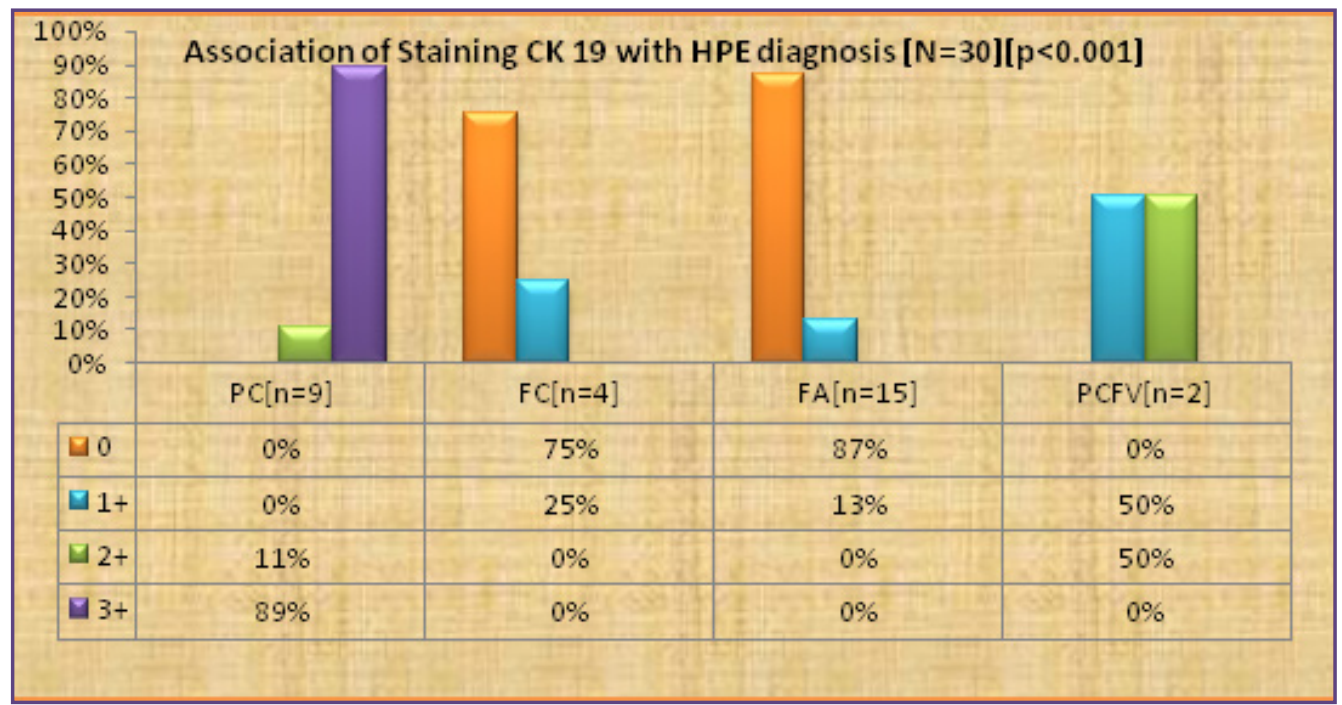

Chart 1: Scoring of Cytokeratin19 Expression in Thyroid Nodules 


\section{Discussion}

Prevalence of thyroid nodules steadily increases with age. Presence of solitary nodule, a multinodular gland and one or more cystic lesions indicate nodular thyroid diseases. ${ }^{[8]}$ But $80 \%$ of the thyroid nodules are benign or hyperplastic nodules. Thyroid malignancies are the most common endocrine carcinomas comprising 5-10\%. ${ }^{[2]}$ Diagnosis of thyroid lesions is difficult when multiple different architectural patterns are present within the same tumor. In addition, encapsulated tumors with follicular pattern pose dilemma in differentiating benign from malignant thyroid neoplasm, when the neoplastic nodule has incomplete capsular invasion or equivocal vascular invasion. ${ }^{[9]}$ Follicular neoplasm/ atypical cells of undetermined significance is yet another category which accounts for $10-25 \%$ of all cases and represents a therapeutic problem because of low risk of malignancy. ${ }^{[10]}$ In view of all these shortcomings, nowadays a panel of immunohistochemical markers are used that help in the distinction of benign and malignant thyroid lesions. Many studies have been done using various immunomarkers to distinguish thyroid nodules but their role in diagnosis is still questionable. In this present study, the usefulness of cytokeratin19 in the differential diagnosis of various thyroid nodules has been analysed and correlated with histomorphology.

Alshenawy et al observed focal and weak cytokeratin 19 positivity in $57 \%$ of follicular adenoma i.e. 4 cases out of total 7 cases in his study. ${ }^{[7]}$ Husain A Saleh et al, in their study reported cytokeratin 19 positivity in $30 \%$ ( 23 cases out of 46 total cases) of cases of follicular adenoma and the intensity of staining is weak and focal. ${ }^{[9]}$ In our present study, the results were similarly weak and focally positive with cytokeratin 19 i.e. $1+$ in 2 cases out of total 15 cases, constituting 13\%. All other 13 cases showed negative staining. Hence cytokeratin19 is not a sensitive marker in the diagnosis of follicular adenoma. Lam et al, reported focal positivity in $68 \%$ of follicular carcinoma, 13 cases out of total 19 cases with cytokeratin19. ${ }^{[1]}$

In this present study we obtained focal $1+$ positivity in only 1 case out of total 4 cases of follicular carcinoma constituting $25 \%$. The sensitivity and specificity of cytokeratin19 in follicular carcinoma in this study was $25 \%$ and $50 \%$ respectively. Demellawy et al observed, cytokeratin 19 positivity in $85 \%$ of cases of papillary carcinoma which included both classic and follicular variant. ${ }^{[12]}$

We studied the cytokeratin 19 staining in total of 11 cases of papillary carcinoma including follicular variant. All the 11 cases showed positive staining $(100 \%)$ with cytokeratin 19 but with varying intensity. 8 cases expressed diffuse and strong positivity of $3+(89 \%)$ in classic papillary carcinoma. Only one case $(11 \%)$ showed $2+$ intensity of staining.

One of the above cases of classic papillary carcinoma had cervical lymph node metastasis and cytokertin 19 showed diffuse and strong $3+$ positivity (Fig 6). Among the 2 cases of follicular variant of papillary carcinoma one showed $1+$ score and other had score $2+$. Similar results were observed in a study by Saleh et al i.e $85 \%$ and Alshenawy found $100 \%$ positive expression of cytokeratin 19 in papillary carcinoma and its follicular variant. ${ }^{[9,7]}$

The sensitivity and specificity of cytokeratin 19 in papillary carcinoma in our study was $100 \%$ and $84 \%$ respectively. Chi square test of these variables showed statistically significant $p$ value of $<0.001$ (Table 4).

The results of this present study are in conformity with the previously conducted studies

(Table 5) and thus immunomarker cytokeratin 19 plays a pivotal role in the differentiation of thyroid nodules.

\section{Conclusion}

From this study cytokeratin 19 is found to be a specific and sensitive $(100 \%)$ marker for papillary carcinoma of thyroid and its follicular variant. The role of this marker in all other variants of papillary carcinoma has to be assessed. The identification of a specific marker for follicular neoplasms is crucial as using a panel of markers can turn out to be a cost effective test for the patient.

\section{Acknowledgements}

I wholeheartedly extend my sincere thanks to my guide Dr. Dhanalakshmi M.D, Associate Professor and Dr. C. Lalitha M.D, Head of the department and Professor, Department of Pathology, Coimbatore medical college for their valuable guidance and support to do this study successfully. I also thank our institution Ethical committee for giving permission to undertake this study.

\section{Reference}

1. Oestreicher-Kedem Y, Halpern M, Roizman P, Hardy B, Sulkes J, Feinmesser R, Stern Y. Diagnostic value of galectin3 as a marker for malignancy in follicular patterned thyroid lesion. Head Neck. 2004; 26(11):960-6.

2. Gilliland F D, Hunt W C, Morris D M et al. 1997 Prognostic factors for thyroid carcinoma. A population-based study of 15,698 cases from the Surveillance, Epidemiology and End Results (SEER) program 1973-1991. Cancer 79: 564-573.

3. Barroeta JE, Baloch ZW, Lal P, Pasha TL, Zhang PJ, LiVolsi VA. Diagnostic value of differential expression of CK19 Galectin-3, HME-1, ERK, RET and p16 in benign and malignant follicular derived lesions of the thyroid- an 
immunohistochemical tissue microarray analysis. Endocr Pathol. 2006; 17(3):225-34.

4. LiVolsi VA. Current concepts in follicular tumors of thyroid. Monogr Pathol. 1993:118-137.

5. Fonseca E, Soares P, Cardoso-Oliveira M, SobrinhoSimões M. Diagnostic criteria in well-differentiated thyroid carcinomas. Endocr Pathol, 2006; 17:109-117.

6. Chan JK. Strict criteria should be applied in the diagnosis of encapsulated follicular variant of papillary thyroid carcinoma. Am J Clin Pathol.2002; 117: 16-18.

7. Alshenawy HA. Utility of immunohistochemical markers in differential diagnosis of follicular cell-derived thyroid lesions. Journal of Microscopy and Ultrastructure. 2014; 2(3): 127-136.

8. Ozolins A, Narbuts Z, Strumfa I, Volanska G, Stepanovs K, Gardovskis J. Immunohistochemical expression of HBME-
1, E-cadherin, and CD56 in the differential diagnosis of thyroid nodules. Medicina (Kaunas). 2012; 48(10): 507-14.

9. Saleh HA., et al. Utility of immunohistochemical markers in differentiating benign from malignant follicular-derived thyroid nodules. Diagnostic Pathology.2010; 5: 9.

10. Fadda G, Rossi ED, Raffaelli M, Pontecorvi A, Sioletic $\mathrm{S}$, Morassi $\mathrm{F}$ et al. Follicular thyroid neoplasms can be classified as low and high risk according to HBME-1 and Galectin-3 expression on liquid based fine needle cytology. Eur J Endocrinol. 2011; 165 (3):447-453.

11. Lam K Y, Lui MC, Lo CY: Cytokeratin expression profiles in thyroid carcinomas. Eur J Surg Oncol. 2001; 27(7): 631-5.

12. Demellawy DE, Nasr A, Alowami S. Application of CD56, p63 and CK19 immunohistochemistry in the diagnosis of papillary carcinoma of the thyroid. Diagn Pathol.2008; 3: 5.

*Corresponding author:

Dr. P. Suriyaprabha, No-53, KVB nagar, 2nd cross, Near vaiyapuri nagar, Karur-639002. Tamilnadu, India.

Phone: +91 9940985464

Email: ptsuri2287@gmail.com

Date of Submission : 30.08.2016

Date of Acceptance : 21.12.2016

Financial or other Competing Interests: None.
Date of Publication : 23.02.2017 